\title{
ANAP

\section{ANÁLISE COMPARATIVA DOS CUSTOS DE CONSTRUÇÃO DA CASA PET E DE UM AMBIENTE CONSTRUÍDO COM MATERIAIS DE ALVENARIA}

\author{
Ana Cláudia Marassá Roza Boso ${ }^{1}$ \\ Camila Pires Cremasco Gabriel $^{2}$ \\ Luís Roberto Almeida Gabriel Filho ${ }^{3}$
}

\begin{abstract}
RESUMO
No gerenciamento do resíduo gerado pelos avanços da humanidade, a reciclagem e a reutilização de materiais descartáveis surge como alternativas para reduzir os impactos ambientais e amenizar as situações dos aterros sanitários quanto o grande espaço ocupado por tais resíduos. Entre esses, o plástico é o material que mais causa danos ambientais, pois apresentam características que dificultam sua degradação. Pensando neste aspecto, a construção civil encontrou uma maneira de substituir matérias de alvenaria por garrafas pet, que por se tratar de um material de alta resistência, se tornaram uma aliada na preservação do meio ambiente através da sustentabilidade. Neste sentido, o presente trabalho tem por finalidade analisar e comparar os custos de construção projeto Casa PET situado no Campus da Fatec de Presidente Prudente - SP e um ambiente com a mesma área construída com materiais de alvenaria.
\end{abstract}

PALAVRAS-CHAVE: Garrafa Pet. Custos de Construção. Substituição.

\section{COMPARATIVE ANALYSIS OF COST PET HOUSE CONSTRUCTION AND A BUILT ENVIRONMENT WITH MASONRY MATERIALS}

\footnotetext{
${ }^{1}$ Faculdades Adamantinenses Integradas, FAI, Adamantina /SP. claudia_boso@hotmail.com.

2 Departamento de Engenharia de Biossistemas da Universidade Estadual Paulista Júlio de Mesquita Filho, UNESP, Campus de Tupã/SP - Brasil. camila@tupa.unesp.br

${ }^{3}$ Departamento de Engenharia de Biossistemas da Universidade Estadual Paulista Júlio de Mesquita Filho, UNESP, Campus de Tupã/SP - Brasil. gabrielfilho@tupa.unesp.br.
} 


\title{
ANAP

\section{RESUME}

The management of waste generated by the advances of humanity, recycling and reuse of disposables emerged as alternatives to reduce environmental impacts and mitigate situations of landfills as the great space occupied by such waste. Among these, plastic is the material that causes more environmental damage, since they have characteristics that hinder their degradation. Thinking about this aspect, the building found a way to replace masonry materials for PET bottles, which in the case of a high strength material, became an ally in preserving the environment through sustainability. In this sense, this paper aims to analyze and compare the project construction costs PET House located on the campus of Fatec of Presidente Prudente $S P$ and an environment with the same area built with masonry

KEYWORDS: Pet Bottle. Construction costs. Replacement.

\section{ANÁLISIS COMPARATIVO DE COSTOS PET CASA CONSTRUCCIÓN Y UN ENTORNO CONSTRUIDA CON MATERIALES DE ALBAÑILERÍA}

\begin{abstract}
RESUMEN
La gestión de los residuos generados por los avances de la humanidad, el reciclaje y la reutilización de materiales desechables surgió como alternativa para reducir los impactos ambientales y mitigar situaciones de vertederos como el gran espacio que ocupa este tipo de residuos. Entre éstos, el plástico es el material que causa daño más del medio ambiente, ya que tienen características que impiden su degradación. Pensando en este aspecto, el edificio se encontró una forma de reemplazar los materiales de albañilería para las botellas de PET, que en el caso de un material de alta resistencia, se convirtió en un aliado en la conservación del medio ambiente a través de la sostenibilidad. En este sentido, el presente trabajo tiene como objetivo analizar y comparar los costos de construcción del proyecto PET Casa situada en el campus de Fatec de Presidente Prudente - SP y un ambiente con la misma área construida con mampostería
\end{abstract}

PALABRAS CLAVE: Botella de PET. Los costos de construcción. Reemplazo.

\section{INTRODUÇÃO}

A degradação do meio ambiente está, em pater, associada ao progresso da humanidade. Um dos problemas causados por esse avanço está na produção de resíduos referentes ao novo modelo de vida da humanidade. Porém esses resíduos trazem uma grande desiquilíbrio físico, químico e biológico ao meio ambiente. O principal e o mais preocupante dos resíduos gerados pela humanidade, esta na degradação causada pelo consumo exagerado de produtos industrializados de fácil descarte (SILVA, A.L.C.; FILHO, L. R. A. G.; CATANEO, P.F., 2015). 


\section{ANAP $B_{\text {rasil }}$ \\ ISSN 1904-3240 \\ v. 8, n. 13}

Segundo Haigert (2009), uma alternativa viável para amenizar os problemas causados por este tipo de produto, esta na reciclagem do mesmo. Com a reciclagem, esses produtos recebem tratamento adequado para reduzir seu volume e amenizar o espaço destinado a este tipo de resíduo em aterros sanitários. Outros benefícios da reciclagem estão no auxilio na renda familiar, na recuperação da matéria-prima, criação de novos postos de trabalho e na reduz dos impactos ambientais.

Outra maneira de amenizar os danos ambientais e a falta de espaço nos aterros sanitários é a reutilização de resíduos industriais. Essa reutilização tem como objetivo aumentar a vida útil do produto sem que passem por processamentos intensos ou por transformações físicas, biológicas e físicoquímicas.

Há inúmeras formas de reutilizar os resíduos industriais, entre elas está à reutilização de produtos recicláveis pela construção civil. O plástico é um desses produtos que gera grandes impactos ambientais, pois apresenta características que dificultam sua degradação. Por ter baixa densidade, ocupar vasto espaço no ambiente e ter um descarte muito rápido, se comparado com outros produtos, o plástico se tornou um grande vilão para locais destinado ao lixo e para locais inapropriados para este tipo de descarte (MANCINI et al., 1998).

O produto mais desenvolvido pelo plástico é as garrafas pet, que são utilizadas para o armazenamento de bebidas e após o seu consumo, são descartadas e destinadas aos aterros sanitários.

Segundo Galli et al. (2012), por se tratar de um material de difícil decomposição e ter demostrado uma grande residência térmica e mecânica, a garrafa pet se tornou uma grande aliada para construção civil pela substituição de materiais de alvenaria. Com essa substituição, os custos de construção se reduzem e consequentemente os impactos ambientais causados por esse produto também se reduz. 


\section{ANAP

Este trabalho tem como objetivo analisar os custos de construção do projeto Casa PET, com os custos de construção de uma área com as mesmas características do projeto, mas desenvolvido com matérias de alvenarias. Neste caso, os materiais de alvenaria utilizados na construção similar ao do projeto são: o tijolo, o bloco de seis furos e o bloco de oito furos.

\section{MATERIAIS E MÉTODOS}

Com o Projeto Casa Pet que foi desenvolvido em 2012 pelos professores Luís Roberto de Almeida Gabriel Filho, Camila Pire Cremasco Gabriel e Daniel dos Santos Viais Neto, pode-se realizar uma comparação dos custos de construção do projeto, com os custos de construção de ambiente com definições idênticas ao projeto Casa PET, mas construída com materiais de alvenaria.

O Projeto Casa Pet situado no Campus da Fatec de Presidente Prudente, tem por finalidade a substituição de materiais de alvenaria por materiais recicláveis. Neste caso, as garrafas pet preenchida com solo-cimento e areia lavada, foram utilizadas como substituição de tijolos ou blocos de cerâmica (GABRIEL, C.P.C.; FILHO, L. R. A. G.; NETO, S. S. V., 2015).

Este projeto baseia-se na construção de uma área de $24 \mathrm{~m}^{2}$, sendo um cômodo com $16 \mathrm{~m}^{2}$ e uma área de serviço com $08 \mathrm{~m}^{2}$, conforme a Figura 1. 


\section{ANAP

Figura1. Planta da Casa PET.

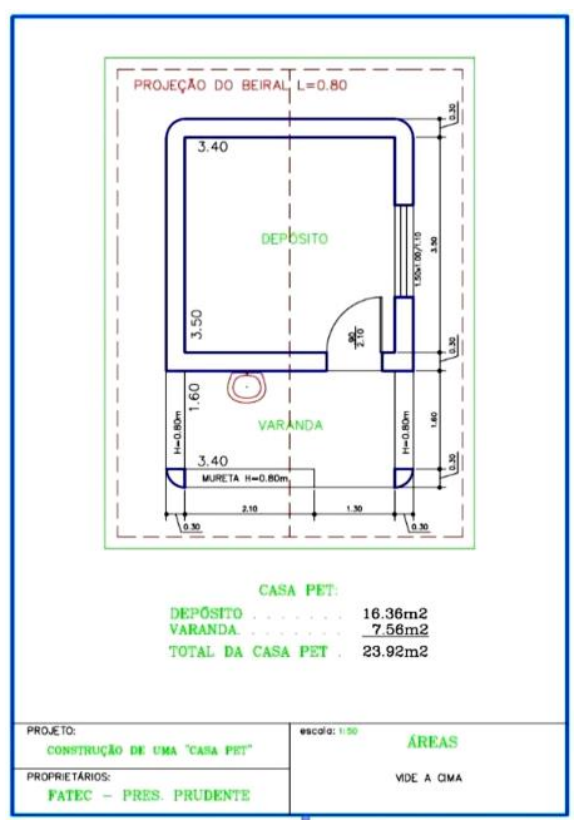

Fonte: Manual de construção da casa PET, 2015.

Foram utilizadas 500 garrafas pet com areia lavada e 3500 garrafas com solo-cimento, sendo esta composta de nove partes de terra vermelha, uma parte de cimento e água para efetuar a mistura.

Para realização da construção da casa pet, foram realizadas as seguintes etapas:

Tabela 1. Etapas da construção da Casa PET.

\begin{tabular}{|c|c|c|}
\hline Etapas & Especificações & Materiais utilizados \\
\hline Baldrame & $\begin{array}{c}30 \mathrm{~cm} \text { de profundidade, } 35 \mathrm{~cm} \\
\text { de largura e comprimento de } \\
\text { acordo com a figura } 1 .\end{array}$ & $\begin{array}{l}\text { Garrafas pet preenchidas } \\
\text { com solo-cimento }\end{array}$ \\
\hline 6 Brocas & $\begin{array}{c}35 \mathrm{~cm} \text { de largura, } 35 \mathrm{~cm} \text { de } \\
\text { comprimento e } 1 \mathrm{~m} \text { de } \\
\text { profundidade. }\end{array}$ & $\begin{array}{l}\text { Concreto e ferro de } \\
\text { construção }\end{array}$ \\
\hline 6 colunas & $\begin{array}{c}\text { Cada coluna foi construída com } \\
2,80 \mathrm{~m}\end{array}$ & $\begin{array}{l}\text { Concreto e ferro de } \\
\text { construção }\end{array}$ \\
\hline Batentes da porta & $\begin{array}{c}90 \mathrm{~cm} \text { de largura por } 2.10 \mathrm{~m} \text { de } \\
\text { comprimento }\end{array}$ & Ferro e concreto \\
\hline Batente da janela & $\begin{array}{c}1,0 \mathrm{~m} \text { de largura por } 1.10 \mathrm{de} \\
\text { comprimento }\end{array}$ & Ferro e concreto \\
\hline
\end{tabular}




\section{ANAP Brasil \\ ISSN 1904-3240 \\ v. 8, n. 13}

REVISTA CIENTIFICA

\begin{tabular}{|c|c|c|}
\hline Paredes & Espessura de $35 \mathrm{~cm}$ & $\begin{array}{l}3500 \text { garrafas de PET } \\
\text { preenchidas com solo- } \\
\text { cimento e massa para } \\
\quad \text { assentar tijolos }\end{array}$ \\
\hline Laje & Área com $38,5 \mathrm{~m}^{2}$ & $\begin{array}{l}\text { Trilhos de concreto com } \\
\text { treliça, placas de isopor e } \\
\text { concreto. }\end{array}$ \\
\hline $\begin{array}{c}\text { Beirais frontais/ Beirais do } \\
\text { fundo }\end{array}$ & $80 \mathrm{~cm} \times 50 \mathrm{~cm} \times 50 \mathrm{~cm}$ & Concreto \\
\hline Fiação elétrica & $\begin{array}{l}\text { Duas luminárias e um } \\
\text { interruptor, uma tomada. }\end{array}$ & $\begin{array}{c}\text { Mangueiras para abrigar a } \\
\text { fiação elétrica, interruptores } \\
\text { e luminárias. }\end{array}$ \\
\hline Contra piso/calçada & De acordo com a figura 1. & Concreto \\
\hline Ligação de agua e esgoto & - & Tubulações \\
\hline $\begin{array}{c}\text { Instalação de Portas e } \\
\text { Janela }\end{array}$ & 01 porta e 01 janela & Ferro \\
\hline Pintura & - & $\begin{array}{c}\text { Tinta acrílica na cor branco } \\
\text { neve e na cor verde folha. } \\
\text { Resina transparente } \\
\text { Tinta esmalte na cor verde } \\
\text { folha } \\
\end{array}$ \\
\hline Piso & & $\begin{array}{c}\text { Argamassa e rejunte na cor } \\
\text { grafite }\end{array}$ \\
\hline Acabamento final & - & $\begin{array}{l}\text { Impermeabilização da parte } \\
\text { superior da laje com } \\
\text { produto específico } \\
\text { Instalação de pedras de } \\
\text { ardósia nas muretas } \\
\end{array}$ \\
\hline Jardim & - & Pedras e árvores \\
\hline
\end{tabular}

Fonte: Gestão ambiental e sustentabilidade, 2015.

Um fator importante do projeto está na projeção da laje. Está sofreu uma preparação especifica para receber uma cobertura verde em futuras construções. Conforme a Figura 2, observa-se a Casa PET finalizada. 


\section{ANAP Brasil \\ ISSN 1904-3240 \\ v. 8, n. 13}

\section{REVISTA C I EN TIF I C A 2015}

Figura 2. Casa PET.

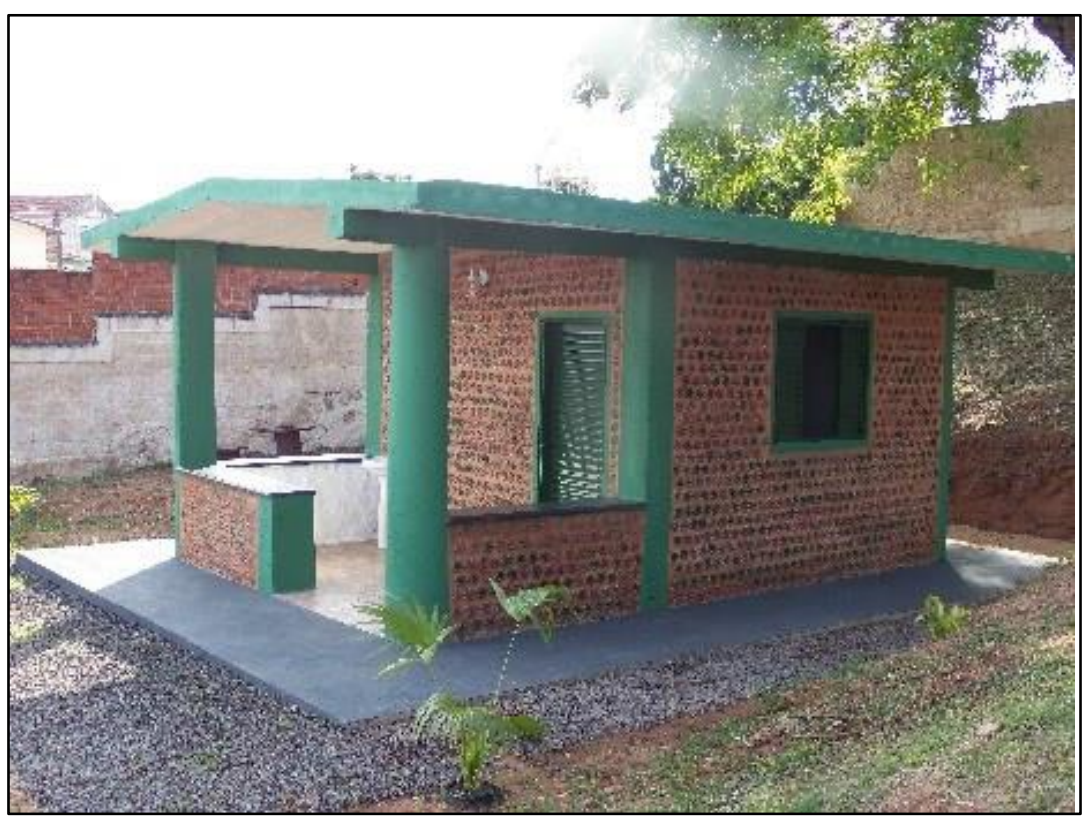

Fonte: Manual de construção da casa PET, 2015.

Com as características do projeto Casa PET, a Figura 3 mostra um quarto de 23,61 $\mathrm{m}^{2}$ de uma residência construído com materiais de alvenaria em 2014.

Figura 3. Ambiente de uma residência construído com materiais de alvenaria.

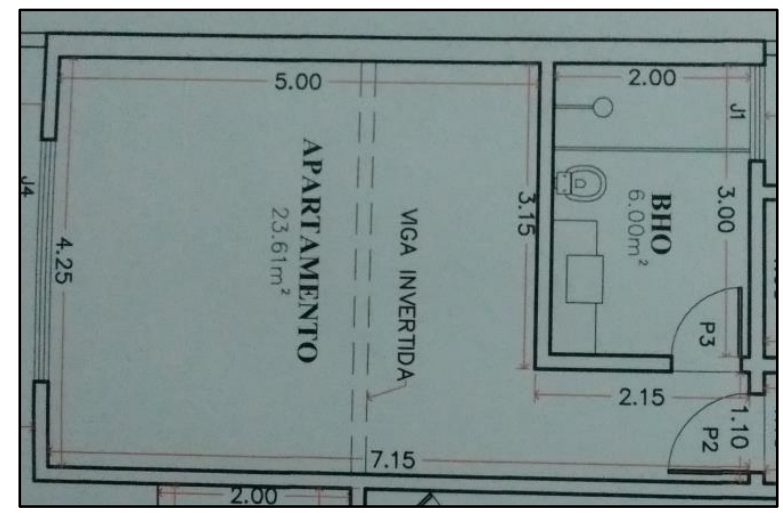

Fonte: Elaborada pelo autor. 


\section{ANAP $B_{\text {rasil }}$ \\ ISSN 1904-3240 \\ v. 8, n. 13}

REVISTA C I ENTIF I CA

Esta construção manteve quase todas as etapas idênticas ao projeto Casa PET, exceto as etapas de iniciação da casa e os materiais utilizados para o levantamento das paredes e no preenchimento da laje.

Assim, o baldrame teve duas fiadas de tijolos com caneleta dupla, quatro ferros de $3 / 8^{\circ}$ conectados com estribo e preenchidos com concreto. Nas brocas foram utilizados ferros de $5 / 16^{\circ}$ conectados com estribo e preenchidos com concreto.

As paredes foram erguidas com blocos de seis furos e com espessura de $15 \mathrm{~cm}$. A laje preenchida com bloco de oito furos e finalizada com concreto.

Para o acabamento interno, foi utilizada a massa corrida na preparação da parede e em seguida tinta látex para finalização do ambiente interno. Para o ambiente externo, foi utilizado o permeabilizaste e a tinta acrílica em seguida.

O termino de ambas as construções se originaram na finalização da pintura e na confecção do jardim envolta dos mesmos.

\section{RESULTADOS}

Para analise do custo de construção da Casa PET, foram considerados apenas os materiais, a mão-de-obra relacionada à construção e mão-de-obra para o preenchimento das garrafas pet. Tabela 2 especifica tais valores:

Tabela 2. Despesas da construção da Casa PET.

\begin{tabular}{cc}
\hline Despesas & Custo Total (R\$) \\
\hline Mão-de-obra (Construção) & $8.405,00$ \\
\hline Materiais & $8.839,11$ \\
\hline Mão-de-obra (preenchimento das garrafas) & $3.120,00$
\end{tabular}

Fonte: Gestão ambiental e sustentabilidade, 2015.

Assim, o custo total da construção da Casa PET foi de $R \$ 20.364,11$, o que equivale à $R \$ 848,50 \mathrm{~m}^{2}$. Mas é pertinente ressaltar que sem os custos de 


\section{ANAP Brasil \\ ISSN 1904-3240 \\ v. 8, n. 13}

REVISTA C I EN TIF I CA

mão-de-obra do preenchimento das garrafas, a construção foi de $R \$ 718,50$ $\mathrm{m}^{2}$.

Com a mesma análise, os custos de construção da área construída com matérias de alvenaria estão demonstrados na Tabela 3.

Tabela3. Despesa de construção de um ambiente com materiais de alvenaria

\begin{tabular}{cc}
\hline Despesas & Custo Total (R\$) \\
\hline Mão-de-obra (Construção) & $8.400,00$ \\
\hline Materiais utilizados na construção & $15.600,00$ \\
\hline
\end{tabular}

Fonte: Elaborado pelo autor.

Portanto, o custo total desta construção é de $R \$ 24.000,00$, o que corresponde à $R \$ 1.000,00 \mathrm{~m}^{2}$.

\section{CONCLUSÕES}

Com analise dos custos de ambas as construções, observa-se que o projeto Casa PET apresentou uma redução significativa no custo total de construção.

Ao se fazer a substituição de materiais de alvenaria por materiais recicláveis, o projeto Casa PET apresentou uma redução de 43,33\% sobre o custo dos materiais utilizados em sua construção.

Outra redução está no custo total da construção do projeto Casa PET. Este obteve uma redução de $15,14 \%$ sobre o custo total da construção do ambiente construído com matérias de alvenaria. O custo total da construção poderá ter um valor maior se considerar a espessura das paredes e colunas, a qualidade e quantidade dos materiais utilizados e o tipo de telhado escolhido para a construção.

O custo da mão de obra de ambas as construções realizados em 2012 e 2014, o que não interferiu nos resultados analisados pelo presente estudo. 


\section{ANAP

Portanto a reutilização de materiais recicláveis apresenta como uma excelente alternativa, no ponto de vista econômico, para redução de resíduos em aterros sanitários e no auxilio para redução dos impactos ambientais.

\section{REFERÊNCIAS BIBLIOGRÁFICAS}

GABRIEL, C.P.C.; FILHO, L. R. A. G.; NETO, S. S. V. Manual de construção da casa PET. Disponível em <http://www.fatecpp.educacao.ws/casapet/manualcasapet.pdf>.Acesso em 05 Set.2015.

FORLIN, F. S.; FARIA, J. A. F. - Reciclagem de embalagens plásticas. Polímeros: Ciência e Tecnologia, vol. 12, no 1, p. 1-10, 2002.

FREIRE, M. T. A.; REYES, F. G. R.; CASTLE, L. Estabilidade térmica de embalagens de poli (tereftalato de etileno (PET): determinação de Oligômeros. Polímeros. vol.8, n.1, 998. p. $46-53$.

GALLI, B; MAGINA, C.; MENEZES, R.; PEREZ, P.; UENO, H. Uso de garrafas de politereftalato de etileno - PET como insumo alternativo na construção de edificações residenciais. In: 1 SNCS - Seminário Nacional de Construções Sustentáveis. IMED. Passo Fundo, 2012.

HAIGERT, F. C. Alternativas para reduzir os impactos socioambientais do descarte de garrafas PET. UFRS. Porto Alegre, 2009.

MANCINI, S. D.; BEZERRA, M. N.; ZANIN, M. Reciclagem de PET advindo de garrafas de refrigerante pós-consumo. Polímeros, 1998, vol.8, no.2, p.68-75.

SANTOS, A. S. F. et al. - Tendências e desafios da reciclagem de embalagens plásticas. Polímeros: Ciência e Tecnologia, vol. 14, n 5, 2004. p. 307-312.

SILVA, A.L.C.; FILHO, L. R. A. G.; CATANEO, P.F. Gestão ambiental e sustentabilidade. Tupã: ANAP, no 1, 2015. p. 145-161. 